\title{
Case Studies, Movies, TV Shows and Other Family Business Resources
}

\author{
Alfredo De Massis (Free University of Bozen-Bolzano) \\ Josip Kotlar (Politecnico di Milano)
}

KEYWORDS: Entrepreneurship, Teaching Methods, Family Business.

The demand for family business education is soaring around the globe, and several higher education institutions are introducing new courses and programs that focus on this dominant type of business organization. Yet, as we discuss in our recent Academy of Management Learning \& Education article (https://journals.aom.org/doi/abs/10.5465/amle.2014.02 46) , universities and teachers have yet to realize the full spectrum of resources available to help them develop and deliver family business education programs.

Current trends in higher education are emphasizing "experiential learning" and technology to enable online, hybrid and collaborative learning. This means that we need to look beyond the traditional textbook and embrace the potential benefits of using a more varied set of resources in the classroom and online learning environments. This motivated us to develop a list that organizes and maps existing learning resources for family business education at undergraduate, masters and executive levels.

Our list includes webinars, television shows, Ted Talks, magazines, movies and case studies. The list is intended to be especially useful for instructors teaching stand-alone family business courses or emphasizing family business issues in other core management courses. It is just a starting point; much much more remains to be done to develop new resources, map resources available around the world, and develop guidelines for their use in the classroom. We invite family business educators to expand and further enhance the pool of learning resources available for family business education.

\section{Acknowledgements}

We would like to thank Carlotta Benedetti, PhD Student at the Free University of Bozen-Bolzano, for her excellent work in helping us to update the lists of movies and teaching cases for family business education. We also gratefully acknowledge the scholars involved in the FERC community for their helpful inputs and feedback. Finally, we are thankful to FamilyBusiness.org for the attention paid to this project, which we feel important for one of the core activities of our mission as family business professors, i.e. the education of the young and senior generations of family business owners and managers.

\section{References}

De Massis, A., Kotlar, J. (2015). Learning resources for family business education: A review and directions for future developments. Academy of Management Learning \& Education, 14(3), 415-422.

\section{On the Small Screen}

Teachers can find webinars, webcasts and short videoclips made available by the Business Families Foundation, the Canadian Association of Family Enterprise and the Family Firm Institute. The BBC series(https://www.bbc.co.uk/programmes/b03q0177) Hidden Histories: Britain's Oldest Family Businesses includes three documentaries about the extraordinary history of Britain's longest-running family businesses. Moreover, teachers can use material from many TV shows such as Arrested Development, The Sopranos, The Sons of Anarchy, and most recently, HBO's Succession, as well as some animated sitcoms such as Bob's Burgers.

Table 1: Selected List of TV Series for Family Business Education
Copyright ( 2019 The Authors. Entrepreneur \& Innovation Exchange is published at EIX.org. This is an open access article under the terms of the Creative Commons Attribution-NoDerivs License, which permits use and distribution in any medium, provided the original work is properly cited and no modifications or adaptations are made. View EIX.org Authorship Terms at https://eix.org/terms
FamilyBusiness 


\begin{tabular}{|c|c|c|c|}
\hline Title & $\begin{array}{l}\text { Starting } \\
\text { Year }\end{array}$ & Creators & Topics \\
\hline Dallas & $1978-1981$ & David Lewis Jacobs & Families, Conflicts \\
\hline The Sopranos & $1999-2007$ & David Chase & Criminal Organization, Leadership, Competition \\
\hline Arrested Development & 2003-2006 & David Schwartz & Decision-making, Stakeholders, Succession, Identity \\
\hline Sons of Anarchy & $2008-2014$ & Kurt Sutter & Brotherhood, Loyalty, Legacy \\
\hline The Good Wife & $2009-2016$ & Robert King, Michelle King & Legal and Political Drama, Network \\
\hline Revenge & $2011-2015$ & Mike Kelley & Identity, Change, Conflicts \\
\hline House of Cards & $2013-2018$ & Beau Willimon & Power, Planning, Lobbyism \\
\hline Empire & 2015-ongoing & Lee Daniels, Danny Strong & Entertainment Industry, Ownership, Succession \\
\hline Dynasty & 2017-ongoing & Richard Alan Shapiro & Succession, Alliances, Competition \\
\hline Family Business & 2018-ongoing & Tony Grounds & Succession, Generations, Leadership \\
\hline Succession & 2018-ongoing & Jesse Armstrong & Entertainment Industry, Ownership, Succession \\
\hline
\end{tabular}




\section{TED Talks}

We also identified some TED talks that can be useful to appreciate and grasp a number of family business topics, as shown in Table 2.

\section{Table 2: Selected List of TED Talks for Family}

\section{Business Education}

\begin{tabular}{|l|l|l|}
\hline Type & Title & Main topic \\
\hline TED global & $\begin{array}{l}\text { Family businesses are here to stay, and thrive } \\
\text { (https://www.ted.com/talks/vikram_bhalla_family_businesses_are_here_to_stay__ } \\
\text { and_thrive) }\end{array}$ & Business growth \\
\hline TEDx Portland & $\begin{array}{l}\text { How doing things differently in a family business can result in success } \\
\text { (https://www.youtube.com/watch?v=0WohxniaPHg) }\end{array}$ & Success \\
\hline TEDx Sayajigunj & $\begin{array}{l}\text { Harnessing the Potential of Family Business } \\
\text { (https://www.youtube.com/watch?v=Z9jzMbyVZV0) }\end{array}$ & Values \\
\hline $\begin{array}{l}\text { TEDx University of } \\
\text { Nevada }\end{array}$ & $\begin{array}{l}\text { Nepotism is my American dream } \\
\text { (https://www.youtube.com/watch?v=Nwpmc1qTMJ4) }\end{array}$ & Nepotism \\
\hline $\begin{array}{l}\text { TEDx King Lincoln } \\
\text { Bronzeville }\end{array}$ & $\begin{array}{l}\text { Transforming lives with vegan family business } \\
\text { (https://www.youtube.com/watch?v=TYwpWjJKL1g) }\end{array}$ & $\begin{array}{l}\text { Business \& } \\
\text { community }\end{array}$ \\
\hline
\end{tabular}




\section{Magazines}

Magazines can be a very valuable resource for family business education. They give students direct access to updated information on current issues relevant to family businesses, as well as many real case examples. Magazines also provide more international perspectives and expert opinions, covering a very broad range of topics and geographical regions. They can be particularly useful for experiential learning. Magazines can be used by educators in a number of ways -- for instance in "blended teaching" initiatives in which a portion of the traditional face-to-face instruction is replaced by learning based on magazine extracts to infuse real-world, practice-oriented learning opportunities into established theory- and notion-based courses.

A growing number of published magazines explicitly focus on family businesses, such as Campden FB (http://www.campdenfb.com/) , Family Business Agenda, Family Business Magazine (https://www.familybusinessmagazine.com/), Tharawat (https://www.tharawat-magazine.com/\#gs.1lopObJ6) magazine and The Practitioner (https://www.thepractitioner.co.uk/) . Moreover, general business magazines increasingly feature family business articles, including Entrepreneur, Business Insider, Business Week, Forbes, The Economist, Wall Street Journal and Washington Post. Finally, teachers can benefit from articles published in blogs and forums with the focus on family business, such as the Family Firm Institute(https://www.ffi.org/) (FFI), Family Business Place(https://familybusinessplace.com/) , Family Business United (http://www.familybusinessunited.com/) , Family Business Network(http://www.fbn-i.org/) (FBN), Family Business Wiki, the International Family Enterprise Research Academy (IFERA), and the Institute for Family Business(https://www.ifb.org.uk/) (IFB) Forum.

\section{On the Big Screen}

Teachers rely increasingly on films in their classes, and their use will become even more frequent as experiential learning continues to grow. Benefits include increasing students' attention and helping them visualize theoretical concepts and issues. This is likely to be particularly valuable in family business courses, as films can depict the many intangible and often sensitive and private aspects of managing family businesses. In our survey of existing courses around the world, we found a broad spectrum of sources for firms that can be used in family business education.

Below is a list of 44 movies that can be useful for engaging students in discussions about family business issues.

Table 3: Selected List of Movies for Family Business Education 


\begin{tabular}{|c|c|c|c|}
\hline Year & Title & Director & Topics \\
\hline 1934 & $\begin{array}{l}\text { The House of } \\
\text { Rothschilds }\end{array}$ & A. L. Werker & $\begin{array}{l}\text { Family Capitalism, International Growth, Legitimacy, Economic } \\
\text { Wealth, Succession }\end{array}$ \\
\hline 1954 & Executive Suite & R. Wise & Change, Governance, Ownership, Strategic Planning, Succession \\
\hline 1956 & The Giant & G. Stevens & $\begin{array}{l}\text { Conflict, In-laws, Ethics, Family Entrepreneurship, Family } \\
\text { Legacy, Succession }\end{array}$ \\
\hline 1958 & $\begin{array}{l}\text { Cat on a Hot Tin } \\
\text { Roof }\end{array}$ & R. Brooks & $\begin{array}{l}\text { Governance, Intergenerational Collaboration, Leadership, } \\
\text { Stakeholder, Succession }\end{array}$ \\
\hline $\begin{array}{l}1972- \\
1990\end{array}$ & $\begin{array}{l}\text { The Godfather } \\
\text { Trilogy }\end{array}$ & F. F.Coppola & $\begin{array}{l}\text { Change, Ethics, Governance, Inheritance, Leadership, Succession, } \\
\text { Values, Culture }\end{array}$ \\
\hline 1984 & This is Spinal Tap & R. Reiner & $\begin{array}{l}\text { Behavior, Conflict, Entrepreneurship, Intra-Senior Generational } \\
\text { Interaction }\end{array}$ \\
\hline 1984 & Places in the Heart & R.Benton & Entrepreneurship, Family-Nonfamily, Stakeholders \\
\hline 1988 & $\begin{array}{l}\text { Tucker: The Man } \\
\text { and His Dream }\end{array}$ & F. F. Coppola & $\begin{array}{l}\text { Goals, Change, Entrepreneurship, Intergenerational Collaboration, } \\
\text { Resources, Strategic Planning, Structure }\end{array}$ \\
\hline 1989 & Family Business & S. Lumet & Altruism, Conflict, Ethics, Family Relations \\
\hline 1991 & Backdraft & R. Howard & Behavior Conflict, Change, Relationship, Siblings \\
\hline 1993 & Rudy & D. Anspaugh & $\begin{array}{l}\text { Entrepreneurship, Evolution, Change, Family Vs. Non-Family } \\
\text { Members, Goals, Inheritance }\end{array}$ \\
\hline 1995 & Sabrina & S. Pollack & $\begin{array}{l}\text { Brother-Brother Relationship, Change, Strategic Planning, } \\
\text { Succession }\end{array}$ \\
\hline 1995 & Tommy Boy & P. Segal & Behavior, Conflict, Goals, Succession \\
\hline 1999 & $\begin{array}{l}\text { Any Given } \\
\text { Sunday }\end{array}$ & O. Stone & Change, Environmental Threats, Goals, Leadership, Succession \\
\hline 2001 & Avalon & M. Oshii & $\begin{array}{l}\text { Change, Governance, Management Development, Network, } \\
\text { Relationship, Strategic Planning, Structure, Structure }\end{array}$ \\
\hline 2001 & Life as a House & I. Winkler & Inter-generational conflict, Illness \\
\hline 2002 & About Schmidt & A. Payne & $\begin{array}{l}\text { Behavior, Conflict, Change, Ownership, Values, Work-Family } \\
\text { Conflict }\end{array}$ \\
\hline 2002 & $\begin{array}{l}\text { My Big Fat Greek } \\
\text { Wedding }\end{array}$ & J.Zwick & $\begin{array}{l}\text { Behavior, Conflict, Culture, Value, Family Involvement, } \\
\text { Ownership }\end{array}$ \\
\hline 2005 & Kinky Boots & J. Jarrold & $\begin{array}{l}\text { Change, Entrepreneurship, Human Resources, Innovation, Social } \\
\text { Capital, Succession }\end{array}$ \\
\hline 2006 & The Queen & S. Frears & $\begin{array}{l}\text { Behavior, Conflict, Change, Environmental Threats, Goals, } \\
\text { Governance, Strategic Planning }\end{array}$ \\
\hline 2007 & $\begin{array}{l}\text { The Will Be } \\
\text { Blood }\end{array}$ & P. T. Anderson & $\begin{array}{l}\text { Entrepreneurship, Competition, Profitability, Business } \\
\text { Opportunities }\end{array}$ \\
\hline 2008 & Bottle Shock & R. Miller & Entrepreneurship, Network, Relationships \\
\hline 2008 & $\begin{array}{l}\text { Mother \& } \\
\text { Daughters }\end{array}$ & C. Bessai & Conflict, Mother-Daughter Relationships \\
\hline
\end{tabular}




\begin{tabular}{|c|c|c|c|}
\hline Year & Title & Director & Topics \\
\hline 2010 & $\begin{array}{l}\text { Romantics } \\
\text { Anonymous }\end{array}$ & J.P. Améris & $\begin{array}{l}\text { Emotions, Entrepreneurship, Family Business Boundaries, } \\
\text { Innovation }\end{array}$ \\
\hline 2010 & Fathers \& Sons & C. Bessai & Conflict, Father-Son Relationships \\
\hline 2010 & $\begin{array}{l}\text { The Social } \\
\text { Network }\end{array}$ & D. Fincher & $\begin{array}{l}\text { Challenges, Entrepreneurship, Resilience, Storytelling, Conflicts, } \\
\text { Success }\end{array}$ \\
\hline 2011 & The Inheritance & P. Fly & $\begin{array}{l}\text { Conflict Management, Family Relations, Goals, Success, } \\
\text { Succession, Work-Family Conflict }\end{array}$ \\
\hline 2011 & Sisters \& Brothers & C. Bessai & Conflict, Sibling Relationships \\
\hline 2011 & Moneyball & B. Miller & $\begin{array}{l}\text { Entrepreneurship, Innovation, Competition, Team Building, } \\
\text { Performance }\end{array}$ \\
\hline 2011 & $\begin{array}{l}\text { Something } \\
\text { Ventured }\end{array}$ & D. Geller, D. Goldfine & Stakeholders, Investments, Innovation \\
\hline 2012 & The Brave & B. Chapman & Intergenerational Conflict, Succession, \\
\hline 2012 & Arbitrage & N. Jarecki & $\begin{array}{l}\text { Parenthood, Altruism, Conflict, Ethics, Family Relations, Power, } \\
\text { Sibling Relationship }\end{array}$ \\
\hline 2013 & Saving Mr. Banks & J. L. Hancock & Change, Identity, Iconic Personality, Creativity, Storytelling \\
\hline 2013 & $\begin{array}{l}\text { The Wolf of Wall } \\
\text { Street }\end{array}$ & M. Scorsese & Goals, Power, Success, Ethics, Behavior and Conflicts \\
\hline 2014 & $\begin{array}{l}\text { A Most Violent } \\
\text { Year }\end{array}$ & J. C. Chandor & $\begin{array}{l}\text { Entrepreneurship, Power, Growth, Competition, Conflicts, } \\
\text { Behaviors, Ethics }\end{array}$ \\
\hline 2015 & Joy & D. O. Russel & Power, Family Relations, Gender, Success, Resilience \\
\hline 2015 & The Intern & N. Meyers & $\begin{array}{l}\text { Gender Stereotypes, Age, Entrepreneurship, Passion, Integrity, } \\
\text { Team Value, CSR, Family Relations }\end{array}$ \\
\hline 2015 & $\begin{array}{l}\text { Unfinished } \\
\text { Business }\end{array}$ & K. Scott & Challenges, Inspiration, Identity, Resilience, Problem-solving \\
\hline 2016 & Adidas VS Puma & O. Dommenget & Brotherhood, Conflicts, Succession, Family Relations \\
\hline 2016 & The Founders & J. L. Hancock & Negotiation Skills, Brotherhood, Storytelling, Growth \\
\hline 2017 & Coco & $\begin{array}{l}\text { L. Unkrich, A. Molina, J. } \\
\text { Katz, M. Aldrich }\end{array}$ & Intergenerational Conflict, Tradition, Legacy \\
\hline 2017 & The Post & S. Spielberg & Succession, Ownership \\
\hline 2018 & $\begin{array}{l}\text { Crazy Rich } \\
\text { Asians }\end{array}$ & J. M. Chu & $\begin{array}{l}\text { Family Relations, Transgenerational Interaction, Family } \\
\text { Relations, Succession, Cultural Conflicts }\end{array}$ \\
\hline 2018 & Lionheart & G. Nnaji & $\begin{array}{l}\text { Change, Ownership, Strategic Planning, Succession, } \\
\text { Intergenerational Collaboration, Leadership, Innovation, Sibling } \\
\text { Relationships, Family Relations }\end{array}$ \\
\hline
\end{tabular}




\section{Teaching Case Studies}

An increasing number of business schools rely on case studies as a primary means for delivering education contents, which emerges as important means to help students link theory and practice. Real-life case studies can help improve students' ability to match theories and models commonly taught in the classroom to actual problem situations, and to learn how to address them. Case studies are likely to be particularly well suited for family business education, as examining the case of "others" offers a privileged opportunity to safely address sensitive and private issues.

In our review, we identified 59 case studies. Table 4 lists these case studies. In order to facilitate search and selection, the table reports the title of each case study, source, industry, geography, firm age, and the topics addressed in each case study. These cases can be used to teach a variety of family business topics to undergraduate, graduate and executive education classes.

\section{Table 4: Selected Case Studies for Family} Business Education 


\begin{tabular}{|c|c|c|c|c|c|}
\hline Case Study & Source & Industry & Geography & $\begin{array}{l}\text { Firm } \\
\text { Age }\end{array}$ & Topic \\
\hline $\begin{array}{l}\text { A Diamond in the Rough: J. M. Huber and the PATH Business } \\
\text { (https://hbsp.harvard.edu/product/KE1002-PDF- } \\
\text { ENG?itemFindingMethod=Search) }\end{array}$ & $\begin{array}{l}\text { Harvard Business } \\
\text { School }\end{array}$ & $\begin{array}{l}\text { Fire retardant additives } \\
\text { business }\end{array}$ & USA & n.a. & $\begin{array}{l}\text { Decision making, Mergers \& } \\
\text { acquisitions, Board of Directors, } \\
\text { Competitive strategy, Risk management, } \\
\text { Growth }\end{array}$ \\
\hline $\begin{array}{l}\text { AAC Technologies (A): Entrepreneurship, Growth and } \\
\text { Transformation(https://hbsp.harvard.edu/product/NTU087-PDF- } \\
\text { ENG?itemFindingMethod=Search) }\end{array}$ & $\begin{array}{l}\text { Harvard Business } \\
\text { School }\end{array}$ & $\begin{array}{l}\text { Manufacturing, } \\
\text { computers \& } \\
\text { electronics, mobile } \\
\text { devices }\end{array}$ & China & n.a. & $\begin{array}{l}\text { Entrepreneurship, Innovation, } \\
\text { Leadership, Technology, Manufacturing, } \\
\text { Growth strategy }\end{array}$ \\
\hline Adams Funeral Home & Poza \& Daugherty & Funerals & Georgia USA & 139 & Strategic Management, Merger analysis \\
\hline $\begin{array}{l}\text { Alchemy of Innovation at TSL Jewellery Ltd. Adding Value to } \\
\text { Gold-Transforming a Traditional Business } \\
\text { (https://hbsp.harvard.edu/product/ST41-PDF- } \\
\text { ENG?itemFindingMethod=Search) }\end{array}$ & $\begin{array}{l}\text { Harvard Business } \\
\text { School }\end{array}$ & Technology, jewelry & Hong Kong & n.a. & $\begin{array}{l}\text { Organizational Perspectives, } \\
\text { Entrepreneurial Management, Innovation, } \\
\text { Change Management, Technology, } \\
\text { Human resource Management, }\end{array}$ \\
\hline Alcoholes de Centroamerica, S.A. & NACRA & Alcohol & Honduras & 46 & $\begin{array}{l}\text { Entrepreneurship, Conflicts Management, } \\
\text { Family Relationship, Strategy }\end{array}$ \\
\hline $\begin{array}{l}\text { Alsea: A New CEO Comes on Board } \\
\text { (https://hbsp.harvard.edu/product/W17441-PDF- } \\
\text { ENG?itemFindingMethod=Search) }\end{array}$ & $\begin{array}{l}\text { Harvard Business } \\
\text { School }\end{array}$ & $\begin{array}{l}\text { Accommodation \& } \\
\text { food services, } \\
\text { restaurants }\end{array}$ & $\begin{array}{l}\text { Spain, Colombia } \\
\text { Mexico }\end{array}$ & n.a. & Franchising, Corporate strategy \\
\hline $\begin{array}{l}\text { Amarnath Gupta and Sons: The Family Business } \\
\text { (https://hbsp.harvard.edu/product/W16702-PDF- } \\
\text { ENG?itemFindingMethod=Search) }\end{array}$ & $\begin{array}{l}\text { Harvard Business } \\
\text { School }\end{array}$ & $\begin{array}{l}\text { Retail trade, petroleum } \\
\text { products }\end{array}$ & India & 66 & Succession Planning, Succession Issues \\
\hline $\begin{array}{l}\text { Amorim: The Future of Natural Cork } \\
\text { (https://hbsp.harvard.edu/product/W17053-PDF- } \\
\text { ENG?itemFindingMethod=Search) }\end{array}$ & $\begin{array}{l}\text { Harvard Business } \\
\text { School }\end{array}$ & $\begin{array}{l}\text { Wine industry, } \\
\text { alcoholic beverages, } \\
\text { manufacturing }\end{array}$ & Portugal & n.a. & $\begin{array}{l}\text { Vertical integration, Packaging, } \\
\text { Manufacturing, Sustainability }\end{array}$ \\
\hline Anderson Steel Service, Inc. & $\begin{array}{l}\text { Harvard Business } \\
\text { School }\end{array}$ & Steel products & Arizona USA & 57 & $\begin{array}{l}\text { Organizational Change, International } \\
\text { Management }\end{array}$ \\
\hline
\end{tabular}




\begin{tabular}{|c|c|c|c|c|c|}
\hline Case Study & Source & Industry & Geography & $\begin{array}{l}\text { Firm } \\
\text { Age }\end{array}$ & Topic \\
\hline Argentina Suites: A Case Study & NACRA & Hotels & Argentina & 26 & $\begin{array}{l}\text { Company Management, Family } \\
\text { Relationships }\end{array}$ \\
\hline $\begin{array}{l}\text { Artecola: Building a Global Brazilian Multinational from Rio } \\
\text { Grande do Sul(https://hbsp.harvard.edu/product/INS941-PDF- } \\
\text { ENG?itemFindingMethod=Search) }\end{array}$ & $\begin{array}{l}\text { Harvard Business } \\
\text { School }\end{array}$ & Adhesives \& sealants & Brazil & n.a. & $\begin{array}{l}\text { Internationalization, Mergers \& } \\
\text { Acquisitions, Emerging markets, } \\
\text { Innovation, Strategic alliances }\end{array}$ \\
\hline $\begin{array}{l}\text { Askew's Foods versus Wal-Mart: Sustainable versus Low-Cost } \\
\text { (https://hbsp.harvard.edu/search?N=\&Nrpp=10\&Ntt=Askew\%2 } \\
\text { 7s+Foods+versus+Wal-Mart\%3A+Sustainable+versus+Low- } \\
\text { Cost) }\end{array}$ & $\begin{array}{l}\text { Harvard Business } \\
\text { School }\end{array}$ & Retail trade & Canada & n.a. & $\begin{array}{l}\text { Corporate Social Entrepreneurship, } \\
\text { Competition, Social Responsibility, } \\
\text { Sustainability }\end{array}$ \\
\hline $\begin{array}{l}\text { ATF, Inc.: Fasteners and Family } \\
\text { (https://hbsp.harvard.edu/product/KEL957-PDF- } \\
\text { ENG?itemFindingMethod=Search) }\end{array}$ & $\begin{array}{l}\text { Harvard Business } \\
\text { School }\end{array}$ & $\begin{array}{l}\text { Automotive parts \& } \\
\text { accessories }\end{array}$ & USA & 37 & $\begin{array}{l}\text { Succession Planning, Management } \\
\text { Styles, Leadership }\end{array}$ \\
\hline $\begin{array}{l}\text { Aurum Furniture and In-Law Management } \\
\text { (https://hbsp.harvard.edu/search?N=\&Nrpp=10\&Ntt=Aurum+F } \\
\text { urniture+and+In-Law+Management) }\end{array}$ & $\begin{array}{l}\text { Harvard Business } \\
\text { School }\end{array}$ & Retail trade & USA & n.a. & $\begin{array}{l}\text { Succession Planning, Family-controlled } \\
\text { Businesses, Conflict, }\end{array}$ \\
\hline Ayala Corporation & $\begin{array}{l}\text { Harvard Business } \\
\text { School }\end{array}$ & $\begin{array}{l}\text { Banking, } \\
\text { telecommunications, } \\
\text { water }\end{array}$ & Philippines & 181 & Future Business Opportunities \\
\hline $\begin{array}{l}\text { Babbitt Ranches: Governance and Strategic Planning in a } \\
\text { Family Business }\end{array}$ & $\begin{array}{l}\text { Harvard Business } \\
\text { School }\end{array}$ & Cattle ranching & Arizona, USA & 127 & $\begin{array}{l}\text { Leadership, Company Management, } \\
\text { Succession, Conflicts }\end{array}$ \\
\hline $\begin{array}{l}\text { Balaji Wafers: Taking the Pepsi Challenge } \\
\text { (https://hbsp.harvard.edu/product/W18606-PDF- } \\
\text { ENG?itemFindingMethod=Search) }\end{array}$ & $\begin{array}{l}\text { Harvard Business } \\
\text { School }\end{array}$ & Manufacturing & India & n.a. & Competition, Strategy, Growth Strategy \\
\hline
\end{tabular}




\begin{tabular}{|c|c|c|c|c|c|}
\hline Case Study & Source & Industry & Geography & $\begin{array}{l}\text { Firm } \\
\text { Age }\end{array}$ & Topic \\
\hline $\begin{array}{l}\text { Beacon Group of Hong Kong: Finding Light in the Shadow } \\
\text { Education Industry(https://hbsp.harvard.edu/product/415082- } \\
\text { PDF-ENG?itemFindingMethod=Search) }\end{array}$ & $\begin{array}{l}\text { Harvard Business } \\
\text { School }\end{array}$ & Technology & Hong Kong & 25 ca. & $\begin{array}{l}\text { Education, Entrepreneurship, } \\
\text { Technology, Human resource } \\
\text { Management, Incentives, Motivation }\end{array}$ \\
\hline Bentington Industries & $\begin{array}{l}\text { Harvard Business } \\
\text { School }\end{array}$ & $\begin{array}{l}\text { Consulting and } \\
\text { engineering services }\end{array}$ & England & 81 & $\begin{array}{l}\text { Family Interaction, organizational } \\
\text { Behavior, Leadership }\end{array}$ \\
\hline $\begin{array}{l}\text { Billionaire's Curse: Gun-based Succession Planning for a } \\
\text { Bangkok Market(https://hbsp.harvard.edu/product/IN1180-PDF- } \\
\text { ENG?itemFindingMethod=Search) }\end{array}$ & $\begin{array}{l}\text { Harvard Business } \\
\text { School }\end{array}$ & Food \& beverage & Thailand & n.a. & Succession Issues, Finance, Economics \\
\hline Bhiwar Enterprises & $\begin{array}{l}\text { Richard Ivey } \\
\text { School of Business }\end{array}$ & $\begin{array}{l}\text { Wholesale trade - non- } \\
\text { durable goods }\end{array}$ & Africa & n.a. & Succession Plan, Conflict Management \\
\hline Borrowing to Grow at Andrews Company & Poza \& Daugherty & Manufacturing & USA & n.a. & Family Business, Succession \\
\hline Caso Conservas Diaz S.A. & $\begin{array}{l}\text { Kellogg School of } \\
\text { Management }\end{array}$ & Agriculture & Spain & n.a. & $\begin{array}{l}\text { Family Relations, Succession, } \\
\text { Shareholders }\end{array}$ \\
\hline Clemens Family Corporation & $\begin{array}{l}\text { Iese Business } \\
\text { School }\end{array}$ & Meat processing & Philadelphia USA & 93 & $\begin{array}{l}\text { Organizational Change, Family Council, } \\
\text { Governance, Succession }\end{array}$ \\
\hline $\begin{array}{l}\text { Consumer Goods Company: A Woman Sailing for Success } \\
\text { (http://sk.sagepub.com/cases/consumer-goods-company-a- } \\
\text { woman-sailing-for-success) }\end{array}$ & Sage Knowledge, & $\begin{array}{l}\text { Retail trade, except of } \\
\text { motor vehicles and } \\
\text { motorcycles }\end{array}$ & Lebanon & n.a. & Succession Strategies, Relationships, \\
\hline $\begin{array}{l}\text { Deep Roots Distillery } \\
\text { (https://hbsp.harvard.edu/product/W16113-PDF- } \\
\text { ENG?itemFindingMethod=Search) }\end{array}$ & $\begin{array}{l}\text { Harvard Business } \\
\text { School }\end{array}$ & $\begin{array}{l}\text { Accommodation \& } \\
\text { food services }\end{array}$ & Canada & 4 & $\begin{array}{l}\text { Accommodation, Break Even Analysis, } \\
\text { Differentiation Strategy, Industry } \\
\text { Analysis, Entrepreneurship }\end{array}$ \\
\hline $\begin{array}{l}\text { DLC Management Corporation: Securing Its Future } \\
\text { (https://hbr.org/product/dlc-management-corporation-securing- } \\
\text { its-future/NA0245-PDF-ENG) }\end{array}$ & NACRA & Real estate & New York USA & 28 & $\begin{array}{l}\text { IPO, Decision-Making, Leadership, } \\
\text { Organization Resources, Ownership, } \\
\text { Control }\end{array}$ \\
\hline Donald Salter Communications, Inc. & $\begin{array}{l}\text { Kellogg School of } \\
\text { Management }\end{array}$ & $\begin{array}{l}\text { Broadcasting, } \\
\text { newspaper publishing }\end{array}$ & Idaho USA & 90 ca. & $\begin{array}{l}\text { Asset Management, Motivation, } \\
\text { Incentives }\end{array}$ \\
\hline
\end{tabular}




\begin{tabular}{|c|c|c|c|c|c|}
\hline Case Study & Source & Industry & Geography & $\begin{array}{l}\text { Firm } \\
\text { Age }\end{array}$ & Topic \\
\hline Ed's Dilemma: Succession Planning at Niagara Paving & $\begin{array}{l}\text { Entrepreneurship: } \\
\text { Theory \& Practice }\end{array}$ & Asphalt paving & $\begin{array}{l}\text { Buffalo, New } \\
\text { York, USA }\end{array}$ & 31 & Succession Planning \\
\hline $\begin{array}{l}\text { Eu Yan Sang: Reviving a Traditional Family Business with } \\
\text { Modern Business Principles }\end{array}$ & $\begin{array}{l}\text { Stanford Business } \\
\text { Case }\end{array}$ & $\begin{array}{l}\text { Traditional Chinese } \\
\text { medicine }\end{array}$ & Singapore & 134 & $\begin{array}{l}\text { Growth, Professionalization, Family } \\
\text { Values }\end{array}$ \\
\hline $\begin{array}{l}\text { Families, Fortunes, and Footwear. Reaching Out to the Fourth } \\
\text { Generation of Brazil's Lupo S.A. }\end{array}$ & $\begin{array}{l}\text { Stanford Business } \\
\text { Case }\end{array}$ & Apparel & Brazil & 94 & Succession, Exit Strategy \\
\hline Ford Motor Company's Value Enhancement Plan & $\begin{array}{l}\text { Harvard Business } \\
\text { School }\end{array}$ & Automotive & Michigan, USA & 110 & $\begin{array}{l}\text { Values, Expectations, Succession } \\
\text { Planning }\end{array}$ \\
\hline Francisco de Narváez at Tía: Selling the Family Business & $\begin{array}{l}\text { Harvard Business } \\
\text { School }\end{array}$ & Retail trade & Argentina & 80 & $\begin{array}{l}\text { Capital Structure, Financial Liquidity, } \\
\text { Business and Shareholder Relations }\end{array}$ \\
\hline From Swords to Ploughshares & INSEAD & Storage and transport & Minnesota, USA & $100 \mathrm{ca}$. & Business Exit \\
\hline Glennon Brothers: Old Dogs Need to Learn New Tricks & $\begin{array}{l}\text { Entrepreneurship } \\
\text { Theory and Practice }\end{array}$ & Sawmilling business & Ireland & $100 \mathrm{ca}$. & $\begin{array}{l}\text { Organizational Life Cycle, Managerial } \\
\text { Style, Business Evolution }\end{array}$ \\
\hline Goodwin Sporting Goods & $\begin{array}{l}\text { Richard Ivey } \\
\text { School of Business } \\
\text { Foundation }\end{array}$ & Retail trade & USA & 66 & $\begin{array}{l}\text { Leadership, Performance Evaluation, } \\
\text { Relationships }\end{array}$ \\
\hline Graham Family and the Washington Post Co. & $\begin{array}{l}\text { Harvard Business } \\
\text { School }\end{array}$ & Newspaper publishing & $\begin{array}{l}\text { District of } \\
\text { Columbia USA }\end{array}$ & 80 & Succession Planning, Conflict Resolution \\
\hline H.D. Chasen Company, Inc. & NACRA & Industrial equipment & USA & 61 & $\begin{array}{l}\text { Interpersonal Communication, } \\
\text { Management Succession, Going Public, } \\
\text { Family Relationships }\end{array}$ \\
\hline Iggy's Bread of the World & $\begin{array}{l}\text { Harvard Business } \\
\text { School }\end{array}$ & Baking \& bakeries & USA & 19 & Succession Planning \\
\hline
\end{tabular}




\begin{tabular}{|c|c|c|c|c|c|}
\hline Case Study & Source & Industry & Geography & $\begin{array}{l}\text { Firm } \\
\text { Age }\end{array}$ & Topic \\
\hline $\begin{array}{l}\text { Ireka Construction Berhad: A Chinese Family Business Goes } \\
\text { Public }\end{array}$ & $\begin{array}{l}\text { Richard Ivey } \\
\text { School of Business } \\
\text { Foundation }\end{array}$ & Construction & Malaysia & 46 & $\begin{array}{l}\text { Growth, Organizational Culture, } \\
\text { Restructuring }\end{array}$ \\
\hline $\begin{array}{l}\text { Is Destira My Destiny? Whether, When, and How to Join One's } \\
\text { Small Family Business } \\
\text { (https://hbsp.harvard.edu/product/KE1048-PDF- } \\
\text { ENG?itemFindingMethod=Search) }\end{array}$ & $\begin{array}{l}\text { Harvard Business } \\
\text { School }\end{array}$ & Apparel & California, USA & 29 & $\begin{array}{l}\text { Succession planning, Decision making, } \\
\text { Entrepreneurship, Compensation, Women } \\
\text { in Business }\end{array}$ \\
\hline $\begin{array}{l}\text { Is This for Me? Career Decision Making in a Family Business } \\
\text { (https://hbsp.harvard.edu/product/NA0463-PDF- } \\
\text { ENG?itemFindingMethod=Search) }\end{array}$ & $\begin{array}{l}\text { Harvard Business } \\
\text { School }\end{array}$ & Colleges \& universities & USA & n.a. & $\begin{array}{l}\text { Human Resource Management, Career } \\
\text { Planning, Personnel Policies, } \\
\text { Generational Issues }\end{array}$ \\
\hline $\begin{array}{l}\text { "It Used to Be a Family Company Until I Joined My Husband } \\
\text { in It": Professionalizing a First-Generation Family Business } \\
\text { (http://sk.sagepub.com/cases/it-used-to-be-a-family-company- } \\
\text { professionalizing-a-family-business) }\end{array}$ & Sage Knowledge, & $\begin{array}{l}\text { Fruits and vegetables } \\
\text { retailing }\end{array}$ & Australia & 48 & Change in Leadership, Leadership Style \\
\hline J. Perez Foods & $\begin{array}{l}\text { Harvard Business } \\
\text { School }\end{array}$ & Food & $\begin{array}{l}\text { Dominican } \\
\text { Republic }\end{array}$ & n.a. & $\begin{array}{l}\text { Business Ownership, Shareholder } \\
\text { Relations, Entrepreneurship, } \\
\text { Organizational problems }\end{array}$ \\
\hline J.R. Pierce, Inc. & NACRA & Pastry & Ohio USA & n.a. & Going public, Change Management \\
\hline $\begin{array}{l}\text { John Nurminen Family: Ownership Strategy Enabling Business } \\
\text { Portfolio Development(http://sk.sagepub.com/cases/john- } \\
\text { nurminen-family-ownership-strategy-portfolio-development) }\end{array}$ & $\begin{array}{l}\text { Sage Knowledge, } \\
2018\end{array}$ & $\begin{array}{l}\text { Transportation and } \\
\text { storage }\end{array}$ & Finland & 148 & $\begin{array}{l}\text { Entrepreneurial Strategies, Ownership } \\
\text { Structure, Business Portfolio } \\
\text { Development, Risk Management, } \\
\text { Opportunity Recognition }\end{array}$ \\
\hline $\begin{array}{l}\text { Keddeg company: Succession to the Next Generation of Small } \\
\text { Business }\end{array}$ & $\begin{array}{l}\text { Kellogg School of } \\
\text { Management }\end{array}$ & Aircraft & USA & 67 & Succession Planning \\
\hline $\begin{array}{l}\text { Ketan Logistics-Charting the Next Route } \\
\text { (https://hbsp.harvard.edu/product/ISB062-PDF- } \\
\text { ENG?itemFindingMethod=Search) }\end{array}$ & $\begin{array}{l}\text { Harvard Business } \\
\text { School }\end{array}$ & $\begin{array}{l}\text { Transportation \& } \\
\text { warehousing }\end{array}$ & India & 33 & $\begin{array}{l}\text { Succession Issues, Entrepreneurship, } \\
\text { Leadership, Stewardship, Corporate } \\
\text { Governance }\end{array}$ \\
\hline
\end{tabular}




\begin{tabular}{|c|c|c|c|c|c|}
\hline Case Study & Source & Industry & Geography & $\begin{array}{l}\text { Firm } \\
\text { Age }\end{array}$ & Topic \\
\hline $\begin{array}{l}\text { Kingston Family Vineyards } \\
\text { (https://hbsp.harvard.edu/product/SM266-PDF- } \\
\text { ENG?itemFindingMethod=Search) }\end{array}$ & $\begin{array}{l}\text { Harvard Business } \\
\text { School }\end{array}$ & Wine industry & Chile & 21 & $\begin{array}{l}\text { Production, Supply Chain Management, } \\
\text { Strategy }\end{array}$ \\
\hline Kohler Co. & $\begin{array}{l}\text { Harvard Business } \\
\text { School }\end{array}$ & Manufacturing & Wisconsin USA & 130 & Conflict Management \\
\hline $\begin{array}{l}\text { K.N. Kordonis \& Co. S.A.: Are We All on the Same Page for } \\
\text { Survival?(http://sk.sagepub.com/cases/kn-kordonis-and-co-sa- } \\
\text { are-we-all-on-the-same-page-for-survival) }\end{array}$ & Sage Knowledge, & Retailing & Greece & n.a. & $\begin{array}{l}\text { Succession Planning, Environmental } \\
\text { Issues, Financial Crisis }\end{array}$ \\
\hline $\begin{array}{l}\text { Leadership Succession at Achal: A Tough Nut to Crack } \\
\text { (https://hbsp.harvard.edu/product/ISB055-PDF- } \\
\text { ENG?itemFindingMethod=Search) }\end{array}$ & $\begin{array}{l}\text { Harvard Business } \\
\text { School }\end{array}$ & Food, agribusiness & India & $40 \mathrm{ca}$. & $\begin{array}{l}\text { Succession Planning, Exit Strategy, } \\
\text { Succession Issues, Valuation, } \\
\text { Entrepreneurship, Leadership Transitions }\end{array}$ \\
\hline $\begin{array}{l}\text { Malkinson Printing Company: The Evolution of a Family } \\
\text { Business(https://hbr.org/product/malkinson-printing-company- } \\
\text { the-evolution-of-a-family-business/NA0347-HCB-ENG) }\end{array}$ & $\begin{array}{l}\text { Harvard Business } \\
\text { School }\end{array}$ & Printing & Wisconsin USA & n.a. & $\begin{array}{l}\text { Organizational Behavior, Conflict, } \\
\text { Generational Issues }\end{array}$ \\
\hline $\begin{array}{l}\text { Managing Strategic Change in Family Business: The Case of } \\
\text { Glopac Chemical(http://sk.sagepub.com/cases/managing- } \\
\text { strategic-change-in-family-business-glopac-chemical) }\end{array}$ & Sage Knowledge, & Chemical & Indonesia & 25 & $\begin{array}{l}\text { Family Conflict, Management Issues, and } \\
\text { Agency Problems }\end{array}$ \\
\hline $\begin{array}{l}\text { Managing to Sell but Failing to Communicate: The Case of the } \\
\text { Family Behind Dow Jones } \\
\text { (http://sk.sagepub.com/cases/managing-to-sell-failing-to- } \\
\text { communicate-family-behind-dow-jones) }\end{array}$ & Sage Knowledge, & Publishing activities & USA & 137 & $\begin{array}{l}\text { Corporate Governance, Strategic } \\
\text { Decision-Making, Professionalization }\end{array}$ \\
\hline $\begin{array}{l}\text { Mehta Engineering: An Indian Family Business Coping With } \\
\text { Growth Through the Third Generation } \\
\text { (http://sk.sagepub.com/cases/mehta-engineering-an-indian- } \\
\text { family-business-coping-with-growth) }\end{array}$ & Sage Knowledge, & $\begin{array}{l}\text { Manufacture of } \\
\text { machinery and } \\
\text { equipment }\end{array}$ & India & $60 \mathrm{ca}$. & $\begin{array}{l}\text { Business Exit, Business Divisions, } \\
\text { Succession Issues }\end{array}$ \\
\hline $\begin{array}{l}\text { Merck, Darmstadt: Sustaining Legacy Beyond } 350 \text { Years } \\
\text { (https://hbsp.harvard.edu/product/ISB123-PDF- } \\
\text { ENG?itemFindingMethod=Search) }\end{array}$ & $\begin{array}{l}\text { Harvard Business } \\
\text { School }\end{array}$ & Pharmaceuticals & Germany & 350 ca. & Governance, Leadership \\
\hline
\end{tabular}




\begin{tabular}{|c|c|c|c|c|c|}
\hline Case Study & Source & Industry & Geography & $\begin{array}{l}\text { Firm } \\
\text { Age }\end{array}$ & Topic \\
\hline Metal Benders Industries, Inc.: The Accidental Entrepreneur & NACRA & Metal industry & Maryland USA & 44 & $\begin{array}{l}\text { Entrepreneurship, Succession, } \\
\text { Shareholder Value, Leadership }\end{array}$ \\
\hline $\begin{array}{l}\text { Monmouth Rubber \& Plastics } \\
\text { (https://hbsp.harvard.edu/product/NA0518-PDF- } \\
\text { ENG?itemFindingMethod=Search) }\end{array}$ & $\begin{array}{l}\text { Harvard Business } \\
\text { School (2018) }\end{array}$ & $\begin{array}{l}\text { Plastics \& rubber } \\
\text { products }\end{array}$ & New Jersey USA & 55 & $\begin{array}{l}\text { Organizational behavior, Decision } \\
\text { Making, Generational Issues }\end{array}$ \\
\hline $\begin{array}{l}\text { Moore Oil: Exit Options for a Multi-Family, Multi- } \\
\text { Generational Small Business } \\
\text { (http://sk.sagepub.com/cases/moore-oil-exit-options-for-multi- } \\
\text { family-generational-small-business) }\end{array}$ & $\begin{array}{l}\text { Sage Knowledge, } \\
2018\end{array}$ & Petroleum marketing & Midwest, USA & 87 & $\begin{array}{l}\text { Entrepreneurial Strategies, Tradition, Exit } \\
\text { Strategies, Ownership, Logistic } \\
\text { Management, Decision-Making }\end{array}$ \\
\hline $\begin{array}{l}\text { Morgan Construction Company: Managing Five Generations of } \\
\text { Family Ownership(http://sk.sagepub.com/cases/morgan- } \\
\text { construction-company-five-generations-family-ownership) }\end{array}$ & $\begin{array}{l}\text { Sage Knowledge, } \\
2018\end{array}$ & $\begin{array}{l}\text { Manufacture of } \\
\text { machine and equipment }\end{array}$ & USA & $120 \mathrm{ca}$. & $\begin{array}{l}\text { Business Exit, Professionalization, } \\
\text { Succession, Intergenerational Dynamics, } \\
\text { Conflict, Decision-Making }\end{array}$ \\
\hline Nantucket Nectars: The Exit & $\begin{array}{l}\text { Harvard Business } \\
\text { School }\end{array}$ & Beverages & USA & 25 & Strategic Management, Leadership \\
\hline Northwest Security Services & $\begin{array}{l}\text { Graduate School of } \\
\text { Business, Stanford } \\
\text { University }\end{array}$ & Security alarm services & USA & 67 & Business Exit \\
\hline $\begin{array}{l}\text { Now What? Now Who? A Mexican Small Family Business in } \\
\text { Transition(http://sk.sagepub.com/cases/now-what-now-who-a- } \\
\text { mexican-small-family-business-in-transition) }\end{array}$ & $\begin{array}{l}\text { Sage Knowledge, } \\
2018\end{array}$ & Carwash & Mexico & 28 & $\begin{array}{l}\text { Conflicts, Succession Planning, Family } \\
\text { Harmony, Business Challenges, } \\
\text { Leadership }\end{array}$ \\
\hline Ottawa Devices, Inc. & $\begin{array}{l}\text { Harvard Business } \\
\text { School }\end{array}$ & $\begin{array}{l}\text { Medical equipment \& } \\
\text { devices }\end{array}$ & Canada & n.a. & Business Exit \\
\hline Outstanding Outsider and the Fumbling Family & $\begin{array}{l}\text { Harvard Business } \\
\text { School }\end{array}$ & Food & Illinois USA & n.a. & $\begin{array}{l}\text { Asset Diversification, Division of } \\
\text { Ownership }\end{array}$ \\
\hline
\end{tabular}




\begin{tabular}{|c|c|c|c|c|c|}
\hline Case Study & Source & Industry & Geography & $\begin{array}{l}\text { Firm } \\
\text { Age }\end{array}$ & Topic \\
\hline $\begin{array}{l}\text { Pho Hoa Dorchester(https://hbsp.harvard.edu/product/317121- } \\
\text { PDF-ENG?itemFindingMethod=Search) }\end{array}$ & $\begin{array}{l}\text { Harvard Business } \\
\text { School (2017) }\end{array}$ & $\begin{array}{l}\text { Food, beverages, } \\
\text { restaurants }\end{array}$ & USA & 27 & $\begin{array}{l}\text { Business Ownership, Change } \\
\text { Management, Organizational Structure, } \\
\text { Organizational Culture, Business Growth, } \\
\text { Leadership }\end{array}$ \\
\hline Pitcairn Family Heritage(R) Fund & $\begin{array}{l}\text { Harvard Business } \\
\text { School }\end{array}$ & Fund investments & PhiladelphiaUSA & 24 & $\begin{array}{l}\text { Change Management, Succession, } \\
\text { Retirement, Conflict of Interests }\end{array}$ \\
\hline Precista Tools AG & $\begin{array}{l}\text { Harvard Business } \\
\text { School }\end{array}$ & Machine tools & Switzerland & 67 & Succession, Family Ownership \\
\hline $\begin{array}{l}\text { Professionalization of Sudarshan Chemical Industries } \\
\text { (https://hbsp.harvard.edu/product/W14727-PDF- } \\
\text { ENG?itemFindingMethod=Search) }\end{array}$ & $\begin{array}{l}\text { Harvard Business } \\
\text { School (2015) }\end{array}$ & Chemical & India & n.a. & $\begin{array}{l}\text { Leadership, Entrepreneurship, Growth } \\
\text { Strategies }\end{array}$ \\
\hline Prudence and Audacity: The House of Beretta & $\begin{array}{l}\text { Harvard Business } \\
\text { School }\end{array}$ & Arms & Brescia, Italy & 487 & Corporate Governance \\
\hline $\begin{array}{l}\text { Rebar Group: A Chinese Family Business Turned Criminal } \\
\text { (http://sk.sagepub.com/cases/rebar-group-a-chinese-family- } \\
\text { business-turned-criminal) }\end{array}$ & $\begin{array}{l}\text { Sage Knowledge, } \\
2018\end{array}$ & $\begin{array}{l}\text { Domestic textiles, } \\
\text { construction, real estate, }\end{array}$ & Taiwan & $60 \mathrm{ca}$. & $\begin{array}{l}\text { Corporate Governance, Corporate } \\
\text { Restructuring, Corporate Fraud }\end{array}$ \\
\hline Salvatore Ferragamo, Spa. & $\begin{array}{l}\text { Harvard Business } \\
\text { School }\end{array}$ & Shoes and leather goods & Italy & 80 ca. & $\begin{array}{l}\text { Family Ownership, Conflicts and } \\
\text { Resolution, Succession }\end{array}$ \\
\hline Sam Steinberg (A) and (B) & $\begin{array}{l}\text { Harvard Business } \\
\text { School }\end{array}$ & $\begin{array}{l}\text { Grocery stores, retail } \\
\text { trade }\end{array}$ & Canada & $100 \mathrm{ca}$. & Business Growth, Start-ups \\
\hline Scholtes Waterservices & $\begin{array}{l}\text { Richard Ivey } \\
\text { School of Business } \\
\text { Foundation }\end{array}$ & Horticultural & Holland & n.a. & Growth, Market Adaptation \\
\hline Sigma Motion, Inc. & $\begin{array}{l}\text { Poza \& Daugherty, } \\
2004\end{array}$ & Linear motion & USA & 38 & $\begin{array}{l}\text { Changes and Strategic Planning, } \\
\text { Corporate Governance, Growth }\end{array}$ \\
\hline Simon Salvage Case: A Case of a Family Business & NACRA & Scrap metal & New Jersey, USA & 68 & $\begin{array}{l}\text { Succession, Governance, } \\
\text { Communication, Leadership }\end{array}$ \\
\hline
\end{tabular}




\begin{tabular}{|c|c|c|c|c|c|}
\hline Case Study & Source & Industry & Geography & $\begin{array}{l}\text { Firm } \\
\text { Age }\end{array}$ & Topic \\
\hline Smit's Company & NACRA & Chocolate & Michigan, USA & 113 & Succession, Conflict Management \\
\hline Stevenson Industries & $\begin{array}{l}\text { Harvard Business } \\
\text { School }\end{array}$ & Pumps \& seals & ConnecticutUSA & 108 & Transition in Ownership, Leadership \\
\hline $\begin{array}{l}\text { Strategy and the Sibling Partnership: The Tan Group from } \\
\text { Indonesia(http://sk.sagepub.com/cases/strategy-and-the-sibling- } \\
\text { partnership-the-tan-group-from-indonesia) }\end{array}$ & $\begin{array}{l}\text { Sage Knowledge, } \\
2018\end{array}$ & Construction company & Indonesia & 70 ca. & $\begin{array}{l}\text { Succession Issues, Growth, Decision- } \\
\text { Making }\end{array}$ \\
\hline Taming My Father & $\begin{array}{l}\text { Sage Knowledge, } \\
2018\end{array}$ & $\begin{array}{l}\text { Manufacturing, } \\
\text { scientific research and } \\
\text { development }\end{array}$ & USA & & $\begin{array}{l}\text { Leadership, Governance, Ownership, } \\
\text { Succession Planning }\end{array}$ \\
\hline The Agnellis and Fiat: Family Business Governance in a Crisis & $\begin{array}{l}\text { Harvard Business } \\
\text { School }\end{array}$ & Automotive & Italy & 113 & $\begin{array}{l}\text { Governance, Leadership, } \\
\text { Professionalization }\end{array}$ \\
\hline The Ambivalent CEO of the Construction Company & $\begin{array}{l}\text { Poza \& Daugherty, } \\
2004\end{array}$ & Construction & USA & n.a. & Growth Strategies \\
\hline $\begin{array}{l}\text { The Business Tycoon, Robert Aston: The Rise and Fall of a } \\
\text { Family Empire(http://sk.sagepub.com/cases/the-business- } \\
\text { tycoon-robert-aston-rise-and-fall-of-a-family-empire) }\end{array}$ & $\begin{array}{l}\text { Sage Knowledge, } \\
2018\end{array}$ & $\begin{array}{l}\text { Accommodation, food } \\
\text { and beverage service } \\
\text { activities }\end{array}$ & Lebanon & n.a. & $\begin{array}{l}\text { Entrepreneurship, Succession Planning, } \\
\text { Diversification Strategies, Organizational } \\
\text { Culture }\end{array}$ \\
\hline The Cousins Tournament Case & $\begin{array}{l}\text { Poza \& Daugherty, } \\
2004\end{array}$ & Electrical systems & California USA & 79 & $\begin{array}{l}\text { Succession, Conflict Management, } \\
\text { Management Style, Organizational } \\
\text { Culture }\end{array}$ \\
\hline The Ferre Media Group (Part A),(Pat B) & $\begin{array}{l}\text { Poza \& Daugherty, } \\
2004\end{array}$ & $\begin{array}{l}\text { News and cement } \\
\text { business }\end{array}$ & Puerto Rico & n.a. & No abstract/key words available \\
\hline The Fojtasek Companies and Heritage partners: March 1995 & $\begin{array}{l}\text { Harvard Business } \\
\text { School }\end{array}$ & Building materials & USA & 70 & No abstract/key words available \\
\hline The Fudge Cottage & NACRA & Confectionery & Michigan, USA & 32 & No abstract/key words available \\
\hline $\begin{array}{l}\text { The Harilela Enterprises: An Indian Family Business in Hong } \\
\text { Kong }\end{array}$ & $\begin{array}{l}\text { Kellogg School of } \\
\text { Management }\end{array}$ & Hotels & Hong Kong & 91 & $\begin{array}{l}\text { Ownership, M\&A, Partnerships, } \\
\text { Succession, Financial Strategy }\end{array}$ \\
\hline
\end{tabular}




\begin{tabular}{|c|c|c|c|c|c|}
\hline Case Study & Source & Industry & Geography & $\begin{array}{l}\text { Firm } \\
\text { Age }\end{array}$ & Topic \\
\hline The Khalil Abdo Group & $\begin{array}{l}\text { Harvard Business } \\
\text { School }\end{array}$ & Pharmaceuticals & Egypt & 67 & Business Exit \\
\hline The New MBA & $\begin{array}{l}\text { Poza \& Daugherty, } \\
2004\end{array}$ & $\begin{array}{l}\text { Construction Sand and } \\
\text { Gravel }\end{array}$ & Minnesota, USA & n.a. & $\begin{array}{l}\text { Ownership, Family Employment, } \\
\text { Management, Leadership, Governance, } \\
\text { Succession }\end{array}$ \\
\hline $\begin{array}{l}\text { The Qadri Group: Innovating in Family Business } \\
\text { (https://hbsp.harvard.edu/product/LCA016-PDF- } \\
\text { ENG?itemFindingMethod=Search) }\end{array}$ & $\begin{array}{l}\text { Harvard Business } \\
\text { School (2012) }\end{array}$ & $\begin{array}{l}\text { Engineering services, } \\
\text { manufacturing }\end{array}$ & Pakistan & n.a. & $\begin{array}{l}\text { Technology Management, Government } \\
\text { Relations }\end{array}$ \\
\hline The Son-In-Law & $\begin{array}{l}\text { Poza \& Daugherty, } \\
2004\end{array}$ & Food (bakery) & USA & 55 & Succession Issues \\
\hline The Wen Group & $\begin{array}{l}\text { Harvard Business } \\
\text { School }\end{array}$ & Pharmaceuticals & Hong Kong & 80 & $\begin{array}{l}\text { Crisis Management, Conflict } \\
\text { Management, Corporate Governance, } \\
\text { Leadership }\end{array}$ \\
\hline $\begin{array}{l}\text { Touchdown Footwear on a Slippery Slope } \\
\text { (https://hbsp.harvard.edu/product/ISB107-PDF- } \\
\text { ENG?itemFindingMethod=Search) }\end{array}$ & $\begin{array}{l}\text { Harvard Business } \\
\text { School (2018) }\end{array}$ & Footwear & India & 54 & $\begin{array}{l}\text { Professionalism, Succession Issues, } \\
\text { Emerging Markets, Leadership, } \\
\text { Stewardship, Corporate Governance, } \\
\text { Organizational Management }\end{array}$ \\
\hline $\begin{array}{l}\text { Trevor Family-Business Compensation Framework: Is It Fair? } \\
\text { (http://sk.sagepub.com/cases/trevor-family-business- } \\
\text { compensation-framework-is-it-fair) }\end{array}$ & $\begin{array}{l}\text { Sage Knowledge, } \\
2018\end{array}$ & Retail trade & Philippines & $50 \mathrm{ca}$. & $\begin{array}{l}\text { Three Circles Model, Ownership, } \\
\text { Conflicts, Compensation Issues }\end{array}$ \\
\hline W.S. Darley \& Co. - Sustaining Family Connections & $\begin{array}{l}\text { Longenecker et al. } \\
\text { (2011) }\end{array}$ & $\begin{array}{l}\text { Fire-fighting and } \\
\text { emergency services }\end{array}$ & Illinois USA & 105 & Succession Planning \\
\hline $\begin{array}{l}\text { Wadeshwar Restaurants: Strategies for Growth } \\
\text { (https://hbsp.harvard.edu/product/ISB107-PDF- } \\
\text { ENG?itemFindingMethod=Search) }\end{array}$ & $\begin{array}{l}\text { Harvard Business } \\
\text { School (2014) }\end{array}$ & $\begin{array}{l}\text { Accommodation \& } \\
\text { food services, } \\
\text { restaurants }\end{array}$ & India & n.a. & Growth Strategy \\
\hline Westbrook Farm & NACRA & Farm & Indiana USA & n.a. & Investment Evaluation, Decision-making \\
\hline
\end{tabular}




\begin{tabular}{|c|c|c|c|c|c|}
\hline Case Study & Source & Industry & Geography & $\begin{array}{l}\text { Firm } \\
\text { Age }\end{array}$ & Topic \\
\hline Weston Nurseries Inc. & $\begin{array}{l}\text { Kellogg School of } \\
\text { Business }\end{array}$ & Florists & USA & 90 & Leadership Strategy \\
\hline $\begin{array}{l}\text { Zandu Pharmaceutical Works: The Takeover Bid } \\
\text { (https://hbsp.harvard.edu/product/ISB045-PDF- } \\
\text { ENG?itemFindingMethod=Search) }\end{array}$ & $\begin{array}{l}\text { Harvard Business } \\
\text { School (2014) }\end{array}$ & Pharmaceuticals & India & $110 \mathrm{ca}$. & $\begin{array}{l}\text { Negotiation, Exit Strategy, Succession } \\
\text { issues, Decision-making, Organizational } \\
\text { Change }\end{array}$ \\
\hline Zobele chemical industries) & $\begin{array}{l}\text { Journal of Business } \\
\text { Venturing }\end{array}$ & Chemical & Italy & 94 & Globalization, Ownership, Control \\
\hline
\end{tabular}


Additional Search Terms: entrepreneurship courses, teaching ideas, teaching resources, classroom ideas, entrepreneurship classes, business schools, business school classes, entrepreneurship students, professors 